OPEN ACCESS

Edited by:

Esther Molina-Montes,

University of Granada, Spain

Reviewed by:

Amir Bagheri,

Tehran University of Medical

Sciences, Iran

Dongfeng Zhang

Qingdao University, China

*Correspondence:

Bo-Chen Pan

panbc@sj-hospital.org

Qi-Jun Wu

wuqj@sj-hospital.org

tThese authors have contributed equally to this work and share first authorship

Specialty section: This article was submitted to Nutritional Epidemiology, a section of the journa

Frontiers in Nutrition

Received: 08 May 2021 Accepted: 06 July 2021

Published: 29 July 2021

Citation:

Liu F-H, Wang X-B, Wen Z-Y, Wang $H-Y$, Zhang $M$, Zhang $S$, Jiang $Y$-T, Zhang J-Y, Sun H, Pan B-C

and Wu Q-J (2021) Dietary Inflammatory Index and Risk of

Asthenozoospermia: A

Hospital-Based Case-Controlled Study in China. Front. Nutr. 8:706869.

doi: 10.3389/fnut.2021.706869

\section{Dietary Inflammatory Index and Risk of Asthenozoospermia: A Hospital-Based Case-Controlled Study in China}

Fang-Hua Liu ${ }^{1,2 \dagger}$, Xiao-Bin Wang ${ }^{3 \dagger}$, Zhao-Yan Wen ${ }^{1,2}$, Han-Yuan Wang ${ }^{1,2}$, Meng Zhang ${ }^{1,2}$, Shuang Zhang ${ }^{1,2}$, Yu-Ting Jiang ${ }^{1,2}$, Jia-Yu Zhang ${ }^{1,2}$, Hui Sun ${ }^{1,2}$, Bo-Chen Pan ${ }^{3 *}$ and Qi-Jun $W u^{1,2 *}$

\begin{abstract}
${ }^{1}$ Clinical Research Center, Shengjing Hospital of China Medical University, Shenyang, China, ${ }^{2}$ Department of Clinical Epidemiology, Shengijing Hospital of China Medical University, Shenyang, China, ${ }^{3}$ Center of Reproductive Medicine, Shengjing Hospital of China Medical University, Shenyang, China
\end{abstract}

Background: Evidence of associations between a pro-inflammatory diet and asthenozoospermia risk is limited. We therefore performed a case-controlled study to investigate associations between pro-inflammatory diet using dietary inflammatory index (DII) scores and asthenozoospermia risk in China.

Methods: Our hospital-based case-controlled study comprised 549 incident asthenozoospermia men and 581 healthy controls. All were interviewed at the infertility clinic in Shengjing Hospital of China Medical University from June 2020 to December 2020. DII scores were calculated based on dietary intake which were assessed using a validated food frequency questionnaire. Semen parameters were analyzed according to World Health Organization guidelines. An unconditional logistic regression model was used to estimate odds ratios (ORs) and corresponding 95\% confidence intervals (Cls) for asthenozoospermia risk. The lowest tertile served as the reference category for regression analyses.

Results: After adjustment for age in the primary multivariable model, we failed to determine a significant negative association between DII and asthenozoospermia risk (for the highest tertile of DII scores compared to the lowest tertile) $(\mathrm{OR}=0.77,95 \% \mathrm{Cl}$ : 0.57-1.03). Similarly, non-significant associations were also identified in the multivariable model after adjusting for more potential confounders ( $\mathrm{OR}=0.86$; $95 \% \mathrm{Cl}$ : 0.58-1.27). Additionally, in subgroup analyses stratified by age, body mass index, and smoking status, non-significant results were consistent with the main findings.

Conclusions: To our knowledge, this is the first study exploring this particular topic. Our research does not support an association between DII scores and asthenozoospermia risk. Further prospective studies with more DII relevant foods and nutrients are warranted to confirm our findings.

Keywords: asthenozoospermia, case-controlled study, dietary inflammatory index, nutrients, China 


\section{INTRODUCTION}

Globally, infertility is a serious public health issue affecting $\sim 15 \%$ of couples of reproductive age $(1,2)$. Notably, male factors account for more than half of all infertility cases $(1,2)$. As a major pathological indicator of male infertility, asthenozoospermia has received considerable attention in recent years. A retrospective study showed that $19 \%$ of infertile males exhibited isolated asthenozoospermia and $63 \%$ of infertile males displayed asthenozoospermia associated with oligoand/or teratozoo-spermia (3). According to a recent Chinese study, the proportion of patients with asthenozoospermia showed an upward trend, accounting for $50.4 \%$ of 38,905 male infertility cases between 2008 and 2016 (4). Equally, emerging evidence has also suggested that genetics (DNAH17 and $A R M C 2$ genes) $(5,6)$, varicocele (7), endocrine-disrupting chemicals (8), stress (9), lifestyle factors such as smoking (10, 11 ), and environmental exposure (12) may also contribute to this condition.

Diet is a major regulator of inflammation and plays important roles in asthenozoospermia occurrence and development (13). Epidemiological studies have shown that adherence to a Western diet pattern is potentially an unfavorable indicator for asthenozoospermia risk (13), whereas Mediterranean dietary patterns are beneficial for sperm motility (14). Western dietary patterns, characterized by a high intake of refined grains, high-fat dairy products, and red meat are associated with a pro-inflammatory state $(15,16)$. In contrast, Mediterranean dietary patterns, characterized by a high intake of green vegetables, fish, whole grains, and fruits, and a low intake of red meat and butter, moderate olive oil consumption, and red wine are associated with an anti-inflammatory state $(17,18)$. Inflammation may be the major cause of the asthenozoospermia (19). Shukla et al. conducted a case-controlled study investigated the association between Tumor Necrosis Factor- $\alpha$ (TNF- $\alpha$ ) (one of inflammatory cytokines) and male infertility in India, and showed that TNF- $\alpha$ were significantly higher in infertile men with asthenozoospermia compared to fertile men (20). To evaluate the potential inflammation impact from various diets, the dietary inflammatory index (DII) was initiated in 2009 and refined in $2014(21,22)$. Based on the literature, the DII accounts for six inflammatory markers; TNF- $\alpha$, Creactive protein, interleukin (IL)-4, IL-6, IL-10, and IL-1 $\beta$, which are related to dietary factors, such as flavonoids, vitamins, macronutrients, minerals, and specific food items (2123). Currently, several epidemiological studies have explored relationships between DII and chronic disease, including metabolic syndrome (24), cardiovascular disease $(25,26)$, and cancer $(27,28)$. These findings have supported the hypothesis that high DII levels are detrimental to health. Surprisingly, only one cross-sectional study involving 209 healthy male college students has investigated the relationship between DII and male infertility risk; with results indicating a positive relationship was identified between DII and total sperm motility and progressive sperm motility in this cohort (29). However, the small sample size and a narrow age group (18-23 years old) may limit the study interpretation.
To provide more evidence on the relationship between DII and asthenozoospermia risk, we conducted a hospital-based casecontrolled study of 549 asthenozoospermia cases and 581 healthy controls in Shenyang, Liaoning Province, China. To the best of our knowledge, ours is the first large-scale study exploring this topic.

\section{MATERIALS AND METHODS Study Design and Participants}

Men admitted to the infertility clinic (Shengjing Hospital of China Medical University) in Liaoning, China were selected for this case-controlled study, conducted between June 2020 and December 2020. Cases included 597 men with asthenozoospermia. According to the fifth edition of the World Health Organization laboratory manual for the examination and processing of human semen (30), asthenozoospermia is defined as "total motility" (progressive + non-progressive) of $<40 \%$, including both rapid and slow progressive motility, slow motility, and non-progressive motility. The condition is also defined as progressive motility of $<32 \%$, including both rapid, slow progressive, and sluggish motility in the same class within $60 \mathrm{~min}$ of ejaculation over the previous 3 months. The total number (or concentration) of spermatozoa and the percentage of morphologically normal spermatozoa is $\geq$ lower reference limits.

Controls comprised of 612 normozoospermic men $(\geq 15 \times$ $10^{6} \mathrm{sperm} / \mathrm{mL}, \geq 40 \%$ total motility, $\geq 32 \%$ progressive motility, and $\geq 4 \%$ normal forms) from infertile couples, admitted to the same infertility clinic with cases group. Patients with a varicocele history were excluded. All participants were interviewed face-toface by trained professional interviewers. Finally, 549 men with asthenozoospermia (92\%) and 581 controls (95\%) completed a validated food frequency questionnaire (FFQ). The research protocol was approved by the ethics committee of Shengjing Hospital of China Medical University.

\section{Semen Collection and Analysis}

Within the required 3-7 days abstinence period, semen samples were collected by masturbation into a plastic tube in a dedicated room. Condoms and lubricants were prohibited. Samples were allowed to liquefy for $45-60 \mathrm{~min}$ before analysis. Sperm is classified into four degrees (A, B, C, D) according to motility by World Health Organization. Ejaculate volume was measured and other parameters such as $\mathrm{pH}$, sperm concentration, total account of sperm, total motility, and the percentage of each motile sperm grade were measured using a WLJY9000 instrument. Flow cytometry was used to assess sperm DNA fragmentation and sperm DNA staining. Pasteurization was used for sperm smear observations and morphology was assessed using an optical microscope. Normal sperm reference values were determined using World Health Organization criteria (30). All analyses were conducted by an experienced technician, and throughout the study, an external quality control was performed.

\section{Data Collection}

Face-to-face interviews were conducted by trained interviewers at baseline using a validated questionnaire which collected 
general demographic characteristics, e.g., health care products (vitamin, zinc, calcium, et al.) and dietary history, personal lifestyle habits, sleep conditions, physical activity, mental condition, passive and indoor smoking, disease history, and family history of chronic disease. Simultaneously, physical examination data were collected, including height, weight [for body mass index (BMI) calculations], waist circumference, hip circumference, and blood pressure.

\section{Exposure Assessment}

Dietary data derived FFQs were used to calculate DII scores for all participants. The DII literature consists of all qualifying publications between 1950 and 2010 reporting one or more associations between dietary components and the following inflammatory markers: IL- $1 \beta$, IL-4, IL-6, IL-10, TNF- $\alpha$, and C-reactive protein (22). In this study, we scored the inflammatory potential for each food parameter according to whether it increased inflammatory or decreased antiinflammatory factors $(+1)$, decreased inflammatory or increased anti-inflammatory factors $(-1)$, or had no effect (0) on inflammatory or anti-inflammatory factors. In the present study, the available components that were used for calculating DII included the following nutrients: energy, carbohydrates, fiber, protein, fat, saturated fatty acids, polyunsaturated fatty acids, monounsaturated fatty acids, anthocyanin, isoflavone, cholesterol, vitamin B12, vitamin B6, beta-carotene, folic acid, niacin, riboflavin, thiamin, vitamin $\mathrm{A}$, vitamin $\mathrm{C}$, vitamin $\mathrm{E}$, zinc, selenium, iron, magnesium, garlic, ginger, onion, pepper and tea. For each participant, in order to compute a $\mathrm{z}$-score, we subtracted the "standard global mean" from the quantity of food items consumed by each subject and divided this by the "global standard deviation" (22). Then, we transformed this value into a centered percentile score to reduce skewness. Finally, we summed DII scores from all foods to calculate an overall DII score. Higher DII scores indicated more a pro-inflammatory diet whereas lower DII scores represented an anti-inflammatory diet.

\section{Statistical Analysis}

Descriptive statistics were performed to profile and compare participant characteristics and food and nutrient intakes between groups. Continuous variables were reported using the mean \pm standard deviation, and categorical variables were represented by counts and percentages. To compare differences between groups, independent sample Student $t$-tests were conducted for continuous variables and the Chi-square test for categorical variables.

Unconditional logistic regression analyses were used to estimate odds ratios (ORs) with 95\% confidence intervals (CIs) to assess associations between DII scores and asthenozoospermia risk. All DII scores were divided into three categories (tertiles), and the lowest tertile of DII scores was considered the reference group. In the first regression model, we only adjusted for age (years). In the second regression model, we further adjusted for BMI $\left(\mathrm{kg} / \mathrm{m}^{2}\right.$ ), smoking status (yes/no), household income (RMB; thousand yuan), abstinence time (days), educational level (junior secondary or below, senior high school/technical secondary school, junior college/university or above), and physical activity
TABLE 1 | General participant characteristics.

\begin{tabular}{|c|c|c|c|}
\hline Characteristics & Asthenozoospermia & Normal & $P$-value \\
\hline No. of participants & 549 & 581 & \\
\hline Age (years) & $33.29 \pm 5.28$ & $32.11 \pm 4.50$ & $<0.05$ \\
\hline Body mass index $\left(\mathrm{kg} / \mathrm{m}^{2}\right)$ & $26.41 \pm 4.42$ & $26.25 \pm 4.55$ & 0.55 \\
\hline $\begin{array}{l}\text { Physical activity } \\
\text { (MET/hours/week) }\end{array}$ & $166.88 \pm 103.42$ & $165.89 \pm 101.80$ & $0 \quad 0.87$ \\
\hline Television watching (hours/week) & $6.52 \pm 8.79$ & $6.19 \pm 8.01$ & 0.52 \\
\hline Computer using (hours/week) & $24.81 \pm 15.95$ & $24.90 \pm 15.07$ & 0.93 \\
\hline Abstinence time (days) & $4.47 \pm 1.48$ & $4.28 \pm 1.39$ & $<0.05$ \\
\hline \multicolumn{4}{|l|}{ Semen parameters } \\
\hline Ejaculate volume (ml) & $3.61 \pm 1.47$ & $3.45 \pm 1.26$ & 0.05 \\
\hline Sperm concentration $\left(10^{6} / \mathrm{ml}\right)$ & $58.74 \pm 36.13$ & $71.10 \pm 39.88$ & $<0.05$ \\
\hline Total sperm count $\left(10^{6} / \mathrm{ml}\right)$ & $198.79 \pm 126.04$ & $232.18 \pm 133.50$ & $0<0.05$ \\
\hline Progress motility (\%) & $22.02 \pm 8.72$ & $44.58 \pm 9.33$ & $<0.05$ \\
\hline Total motility (\%) & $27.94 \pm 10.92$ & $54.94 \pm 11.35$ & $<0.05$ \\
\hline $\begin{array}{l}\text { Normal sperm morphology } \\
\text { (\%) }\end{array}$ & $5.70 \pm 2.55$ & $6.68 \pm 2.72$ & $<0.05$ \\
\hline Smoke status $(n, \%)$ & & & 0.11 \\
\hline No & $285(51.91)$ & $274(47.16)$ & \\
\hline Yes & 264 (48.09) & $307(52.84)$ & \\
\hline Educational level $(n, \%)$ & & & 0.67 \\
\hline Junior secondary or below & $121(22.04)$ & $141(24.27)$ & \\
\hline $\begin{array}{l}\text { Senior high school/technical } \\
\text { secondary school }\end{array}$ & 79 (14.39) & $82(14.11)$ & \\
\hline $\begin{array}{l}\text { Junior college/university or } \\
\text { above }\end{array}$ & $349(63.57)$ & 358 (61.62) & \\
\hline $\begin{array}{l}\text { Annual family income (RMB } \\
\text { thousand yuan), ( } n, \%)\end{array}$ & & & 0.76 \\
\hline$<50$ & $98(17.85)$ & $94(16.18)$ & \\
\hline 50 to $<100$ & 209 (38.07) & $226(38.90)$ & \\
\hline$\geq 100$ & $242(44.08)$ & 261 (44.92) & \\
\hline
\end{tabular}

MET, metabolic equivalent.

All continuous variables are represented as the mean \pm standard deviation. All categorical variables are represented as counts and percentages. $P$-values were derived from Student t-tests and Chi-square tests. All statistical tests are two sided.

(metabolic equivalent/hours/week). In addition, we calculated adjusted risk estimates for asthenozoospermia by DII score in subgroup analyses stratified by age, BMI, and smoking status. All analyses were conducted using SAS version 9.4 (SAS Institute Inc., Cary, NC, USA). All statistical tests were two-sided and statistical significance was set at $P<0.05$.

\section{RESULTS}

The general distribution characteristics of asthenozoospermia between groups are shown (Table 1). Asthenozoospermia cases tended to be slightly older and experience a longer abstinence time than controls. In terms of semen parameters, asthenozoospermia cases had significantly lower sperm concentrations, total sperm counts, progress motility, total motility, and normal sperm morphology than controls.

Food and nutrient intake distributions across groups are also shown (Table 2). When compared with controls, cases displayed 
TABLE 2 | Dietary intake of participants.

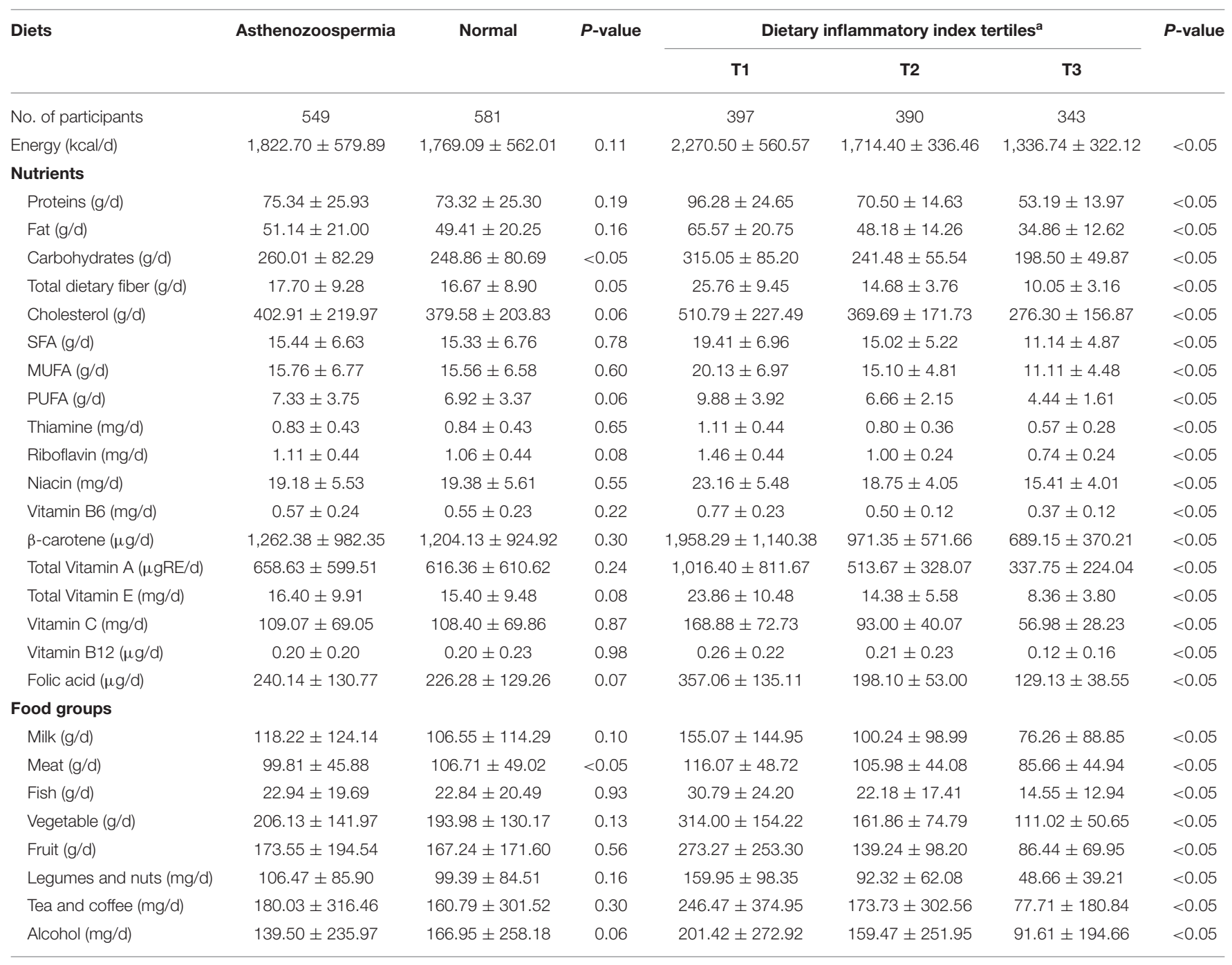

MUFA, monounsaturated fatty acids; PUFA, polyunsaturated fatty acids; SFA, saturated fatty acids.

a Tertile DII score range: $T 1(<0.65)$, T2 $(0.65$ to $<2.32)$, and T3 $(\geq 2.32)$.

All continuous variables are shown as the mean and standard deviation.

All statistical tests are two sided.

higher carbohydrate consumption, however, meat consumption in cases was significantly $(P<0.05)$ lower than controls. Also, dietary intakes of study participants across the DII score tertiles are presented in Table 2 . Significantly decreasing trends were observed for the food and nutrient intake $(P<0.05)$.

Associations between DII scores and asthenozoospermia risk are shown (Table 3). We observed a significant negative association between DII score and the odds of asthenozoospermia in the model 1 (OR $=0.74$, 95\% CI: $0.55-$ 0.98). However, in model 2 which adjusted for age only, the highest DII tertile was not associated with asthenozoospermia risk $(\mathrm{OR}=0.77,95 \% \mathrm{CI}: 0.57-1.03)$ when compared with the lowest tertile. Similar results were also found $\left(\mathrm{OR}_{\mathrm{T} 3 \mathrm{vs} . \mathrm{T} 1}=0.86\right.$, 95\% CI: 0.58-1.27) when further adjusted for BMI, smoking status, household income, total energy intake, abstinence time, educational level, and physical activity. In addition, we evaluated the effect-modifying roles of age, BMI, and smoking status. Not surprisingly, the results of these subgroup analyses were agreed with the main findings (Table 4).

\section{DISCUSSION}

To the best of our knowledge, ours is the first study to investigate the relationship between a pro-inflammatory diet, measured the DII scores, and asthenozoospermia risk. In this hospitalbased case-controlled study, although the point estimate of multivariable regression analyses indicated a negative association between DII scores and asthenozoospermia risk, we observed no statistically significant findings. It might be lack of common components that used to calculate DII (such as n-3 fatty acids, n-6 fatty acids and vitamin D). However, we failed to get the information from the FFQ used in current investigation. 
Moreover, in a hospital-based case-controlled study, recall capabilities and selection bias may influence the accuracy of exposure.

DII studies in asthenozoospermia are scarce. A cross-sectional Spanish study explored the relationship between DII and male reproductive parameters (29). This study in 209 healthy male university students reported that a high DII was positively associated with total sperm motility and progressive sperm motility (29). These Spanish data were inconsistent with our findings, which may be attributed to different participants, habits, outcomes and sample size. Participants included in our study aged 18-79 years, however, the participants of Spanish research are college students and age of population is narrow. In addition, Spain belongs to the Mediterranean region, and the dietary habits may be close to the mediterranean diet which is an anti-inflammatory diet (17). Our study carried out

TABLE 3 | Adjusted risk estimates for asthenozoospermia by dietary inflammatory index.

\begin{tabular}{lcccc}
\hline & \multicolumn{3}{c}{ Dietary inflammatory index tertiles } & \multirow{2}{*}{$\boldsymbol{P}$ for trend } \\
\cline { 2 - 4 } & T1 & T2 & T3 & \\
\hline DIl score range & $<0.65$ & 0.65 to $<2.32$ & $\geq 2.32$ & \\
Cases/Controls & $203 / 193$ & $195 / 193$ & $151 / 195$ & \\
Model 1 & $1.00($ Ref $)$ & $0.96(0.73-1.27)$ & $0.74(0.55-0.98)$ & 0.06 \\
Model 2 & $1.00($ Ref $)$ & $0.97(0.73-1.28)$ & $0.77(0.57-1.03)$ & 0.10 \\
Model 3 & $1.00($ Ref) & $1.05(0.76-1.45)$ & $0.86(0.58-1.27)$ & 0.53 \\
\hline
\end{tabular}

Cl, confidence interval; OR, odds ratio; T, Tertiles; Ref, reference.

a Model 1: unadjusted.

${ }^{\text {b} M o d e l ~ 2: ~ a d j u s t e d ~ f o r ~ a g e . ~}$

${ }^{c}$ Model 3: adjusted for age, body mass index, smoking status, household income, total energy intake, abstinence time, educational level, and physical activity. in Liaoning, China and the dietary habits may be similar to the western dietary patterns of pro-inflammatory diets (15). In addition, two previous studies investigated the relationship between dietary patterns and asthenozoospermia. Eslamian et al. performed two case-controlled studies (107 asthenozoospermic men and 235 age-matched controls) to explore the association between dietary patterns and nutrient patterns and the odds of asthenozoospermia, respectively $(13,31)$. And their findings suggested that western pattern of pro-inflammatory state is positively correlated with asthenozoospermia and pattern comprising mainly of antioxidant nutrients of anti-inflammatory may be inversely associated with asthenozoospermia. The reason for the different results from ours may be due to different food items, such as grains, condiments, sauces and calcium included in previous two studies, but not included in our study.

A previous study showed that inflammation may affect prostate function, which in turn affects sperm quality (32). Researchers also reported increased leukocytosis, IL-1 levels, and reactive oxygen species in patients with chronic prostatitis, along with decreased sperm quality $(33,34)$. Although reproductive tract infections and elevated inflammatory factor levels are associated with negative consequences for male reproduction, spermatogenesis, and semen quality (35), no research has yet uncovered the precise biological mechanisms underpinning DII and asthenozoospermia, therefore future studies in this area are warranted.

This study had several strengths. First, ours was the first report to focus on associations between DII and asthenozoospermia risk. Second, our large sample size and high participation rates were advantageous in reducing random errors often associated with studies with low participant numbers. Furthermore, a validated FFQ was used as a comprehensive method to collect detailed data about habitual food intake associated with inflammatory characteristics. In addition, DII was used as a

TABLE 4 | Adjusted risk estimates for asthenozoospermia by dietary inflammatory index stratified by age, body mass index, and smoking status.

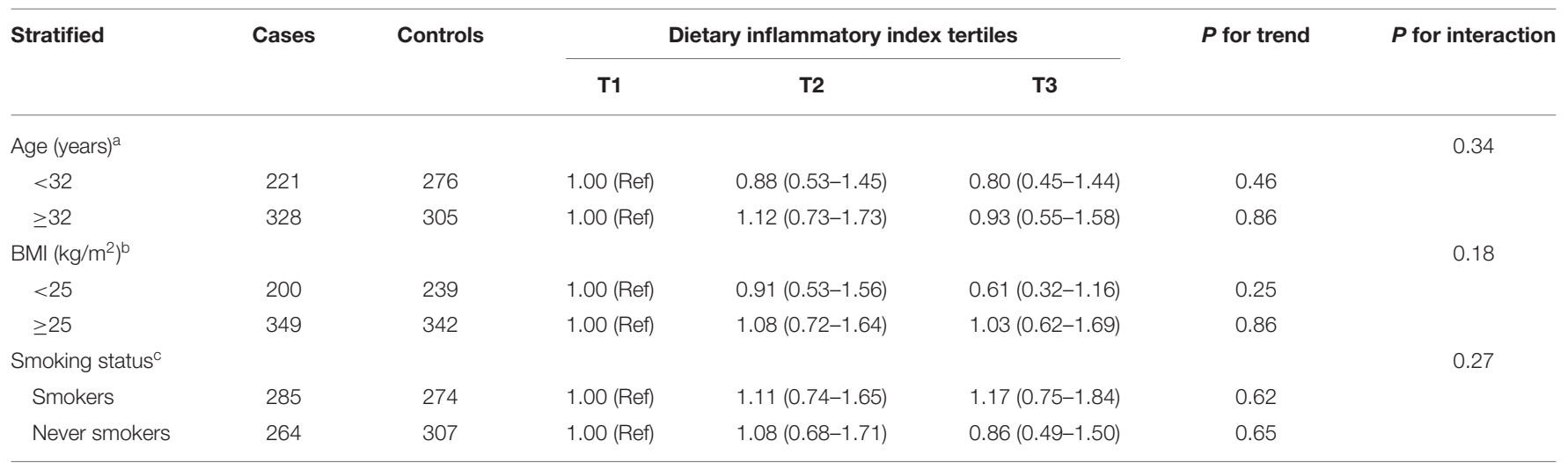

Cl, confidence interval; OR, odds ratio; T, Tertiles; Ref, reference.

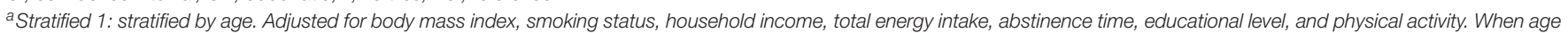
$<32$, the tertile DIl score range is T1 $(<0.87)$, T2 (0.87 to $<2.34)$, and T3 $(\geq 2.34)$. When age $\geq 32$, the tertile DII score range is T1 $(<0.53)$, T2 (0.53 to <2.15), and T3 ( $\geq 2.15)$.

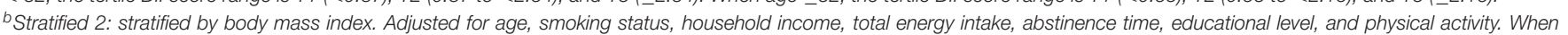

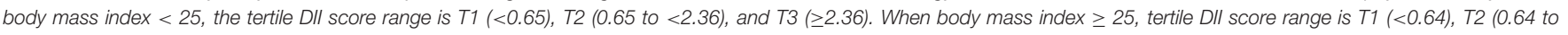
$<2.20)$, and $T 3(\geq 2.20)$.

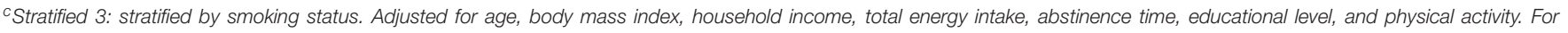
smokers, the tertile DIl score range is $T 1(<0.65)$, T2 (0.65 to <2.37), and T3 ( $\geq 2.37)$. For non-smokers, the tertile DII score range is $T 1(<0.56)$, T2 (0.56 to <2.21), and T3 ( $\geq 2.21)$. 
research tool to focus on the effects of the overall diet on asthenozoospermia risk, instead of individual foods or nutrients. Of note, we adjusted for several confounding factors which ensured the authenticity and reliability of our research results.

Conversely, our study also had several limitations meaning our results must be interpreted with caution. First, as in any hospital-based case-controlled study, recall capabilities and selection bias are problematic. There may have been bias in the accuracy and completeness of case recall of exposure history in the year prior to their diagnosis. Similarly, the control group from the hospital may have made different dietary choices when compared with the general population (36). To improve subject comparability, we used a highly reproducible validated FFQ and selected highly trained and skilled researchers to collect dietary information $(37,38)$. Second, as observed in previous studies (39), we lacked data on some dietary DII components, including flavonoids, turmeric, rosemary, saffron, and thyme, which were not calculated for DII scores; however, as they are uncommon ingredients in Chinese diets, they may have had no significant effects on DII scores. Third, although the FFQ is commonly used to assess food intake in epidemiological studies, measurement bias remains a possibility. Based on standard food portions to calculate food intake, we were unable to determine the precise food intake of participants. Thus, associations between DII and asthenozoospermia risk may be underestimated. To reduce bias effects, energy adjustments were applied to our analyses. Fourthly, although a diverse array of potential confounders was adjusted for in this research, residual confounders cannot be completely excluded, including endocrine-disrupting chemicals (8) and ambient air pollution (40). Future studies should aim to eliminate these issues and clarify the relationship between exposure and outcomes.

In conclusion, our data suggested DII was not associated with asthenozoospermia risk. Future studies must include more food parameters to comprehensively calculate DII scores. Equally,

\section{REFERENCES}

1. Salas-Huetos A, Bullo M, Salas-Salvado J. Dietary patterns, foods and nutrients in male fertility parameters and fecundability: a systematic review of observational studies. Hum Reprod Update. (2017) 23:371-89. doi: 10.1093/humupd/dmx006

2. Agarwal A, Mulgund A, Hamada A, Chyatte MR. A unique view on male infertility around the globe. Reprod Biol Endocrinol. (2015) 13:37. doi: 10.1186/s12958-015-0032-1

3. Curi SM, Ariagno JI, Chenlo PH, Mendeluk GR, Pugliese MN, Sardi SL, et al. Asthenozoospermia: analysis of a large population. Arch Androl. (2003) 49:343-9. doi: 10.1080/01485010390219656

4. Wu ZG, Chen WK, Fei QJ, Liu YL, Liu XD, Huang H, et al. Analysis of semen quality of 38905 infertile male patients during 2008-2016 in Wenzhou, China. Asian J Androl. (2021) 23:314-18. doi: 10.4103/aja.aja_83_20

5. Zhang B, Ma H, Khan T, Ma A, Li T, Zhang H, et al. A DNAH17 missense variant causes flagella destabilization and asthenozoospermia. $J$ Exp Med. (2020) 217. doi: 10.1084/jem.20182365

6. Coutton C, Martinez G, Kherraf ZE, Amiri-Yekta A, Boguenet M, Saut A, et al. Bi-allelic mutations in ARMC2 lead to severe asthenoteratozoospermia due to sperm flagellum malformations in humans and large prospective studies should be designed and implemented to validate our results or identify precise associations between the diet and asthenozoospermia.

\section{DATA AVAILABILITY STATEMENT}

The original contributions presented in the study are included in the article/supplementary material, further inquiries can be directed to the corresponding author/s.

\section{ETHICS STATEMENT}

The studies involving human participants were reviewed and approved by the ethics committee of Shengjing Hospital of China Medical University. The patients/participants provided their written informed consent to participate in this study.

\section{AUTHOR CONTRIBUTIONS}

Q-JW and B-CP conceived the study. F-HL, X-BW, and Z-YW contributed to the design. F-HL, Z-YW, H-YW, and MZ collected the data. SZ and J-YZ cleaned the data and checked the discrepancy. F-HL and SZ analyzed the data. Y-TJ, J-YZ, and HS interpreted the data. All authors interpreted the data, read the manuscript, and approved the final vision.

\section{FUNDING}

This work was supported by 345 Talent Project to Q-JW.

\section{ACKNOWLEDGMENTS}

We thank International Science Editing for editing this manuscript. mice. Am J Hum Genet. (2019) 104:331-40. doi: 10.1016/j.ajhg.2018. 12.013

7. Kurtz MP, Zurakowski D, Rosoklija I, Bauer SB, Borer JG, Johnson KL, et al. Semen parameters in adolescents with varicocele: association with testis volume differential and total testis volume. J Urol. (2015) 193(5 Suppl.):18437. doi: 10.1016/j.juro.2014.10.111

8. Bloom MS, Whitcomb BW, Chen Z, Ye A, Kannan K, Buck LG. Associations between urinary phthalate concentrations and semen quality parameters in a general population. Hum Reprod. (2015) 30:2645-57. doi: 10.1093/humrep/dev219

9. Janevic T, Kahn LG, Landsbergis P, Cirillo PM, Cohn BA, Liu X, et al. Effects of work and life stress on semen quality. Fertil Steril. (2014) 102:530-8. doi: 10.1016/j.fertnstert.2014.04.021

10. Wegner CC, Clifford AL, Jilbert PM, Henry MA, Gentry WL. Abnormally high body mass index and tobacco use are associated with poor sperm quality as revealed by reduced sperm binding to hyaluronan-coated slides. Fertil Steril. (2010) 93:332-4. doi: 10.1016/j.fertnstert.2009.07.970

11. Ramlau-Hansen $\mathrm{CH}$, Thulstrup AM, Aggerholm AS, Jensen MS, Toft G, Bonde JP. Is smoking a risk factor for decreased semen quality? A cross-sectional analysis. Hum Reprod. (2007) 22:188-96. doi: 10.1093/humrep/del364 
12. Zhou Y, Meng T, Wu L, Duan Y, Li G, Shi C, et al. Association between ambient temperature and semen quality: a longitudinal study of 10802 men in China. Environ Int. (2020) 135:105364. doi: 10.1016/j.envint.2019.105364

13. Eslamian G, Amirjannati N, Rashidkhani B, Sadeghi MR, Baghestani AR, Hekmatdoost A. Adherence to the Western pattern is potentially an unfavorable indicator of asthenozoospermia risk: a case-control study. J Am Coll Nutr. (2016) 35:50-8. doi: 10.1080/07315724.2014.936983

14. Salas-Huetos A, Babio N, Carrell DT, Bullo M, Salas-Salvado J. Adherence to the Mediterranean diet is positively associated with sperm motility: a crosssectional analysis. Sci Rep. (2019) 9:3389. doi: 10.1038/s41598-019-39826-7

15. Khayyatzadeh SS, Moohebati M, Mazidi M, Avan A, Tayefi M, Parizadeh SM, et al. Nutrient patterns and their relationship to metabolic syndrome in Iranian adults. Eur J Clin Invest. (2016) 46:840-52. doi: 10.1111/eci.12666

16. King DE, Egan BM, Geesey ME. Relation of dietary fat and fiber to elevation of C-reactive protein. Am J Cardiol. (2003) 92:1335-9. doi: 10.1016/j.amjcard.2003.08.020

17. Estruch R, Martinez-Gonzalez MA, Corella D, Salas-Salvado J, RuizGutierrez V, Covas MI, et al. Effects of a Mediterranean-style diet on cardiovascular risk factors: a randomized trial. Ann Intern Med. (2006) 145:111. doi: 10.7326/0003-4819-145-1-200607040-00004

18. Johansson-Persson A, Ulmius M, Cloetens L, Karhu T, Herzig KH, Onning G. A high intake of dietary fiber influences C-reactive protein and fibrinogen, but not glucose and lipid metabolism, in mildly hypercholesterolemic subjects. Eur J Nutr. (2014) 53:39-48. doi: 10.1007/s00394-013-0496-8

19. Tronchon V, Vialard F, El SM, Dechaud H, Rollet J, Albert M, et al. Tumor necrosis factor-alpha-308 polymorphism in infertile men with altered sperm production or motility. Hum Reprod. (2008) 23:2858-66. doi: 10.1093/humrep/den277

20. Shukla KK, Agnihotri S, Gupta A, Mahdi AA, Mohamed EA, Sankhwar SN, et al. Significant association of TNFalpha and IL- 6 gene with male infertilityan explorative study in Indian populations of Uttar Pradesh. Immunol Lett. (2013) 156:30-7. doi: 10.1016/j.imlet.2013.08.011

21. Cavicchia PP, Steck SE, Hurley TG, Hussey JR, Ma Y, Ockene IS, et al. A new dietary inflammatory index predicts interval changes in serum high-sensitivity C-reactive protein. J Nutr. (2009) 139:2365-72. doi: 10.3945/jn.109.114025

22. Shivappa N, Steck SE, Hurley TG, Hussey JR, Hebert JR. Designing and developing a literature-derived, population-based dietary inflammatory index. Public Health Nutr. (2014) 17:1689-96. doi: $10.1017 /$ S1368980013002115

23. Shivappa N, Steck SE, Hurley TG, Hussey JR, Ma Y, Ockene IS, et al. A population-based dietary inflammatory index predicts levels of C-reactive protein in the Seasonal Variation of Blood Cholesterol Study (SEASONS). Public Health Nutr. (2014) 17:1825-33. doi: 10.1017/S1368980013002565

24. Neufcourt L, Assmann KE, Fezeu LK, Touvier M, Graffouillere L, Shivappa $\mathrm{N}$, et al. Prospective association between the dietary inflammatory index and metabolic syndrome: findings from the SU.VI.MAX study. Nutr Metab Cardiovasc Dis. (2015) 25:988-96. doi: 10.1016/j.numecd.2015. 09.002

25. Garcia-Arellano A, Ramallal R, Ruiz-Canela M, Salas-Salvado J, Corella $\mathrm{D}$, Shivappa $\mathrm{N}$, et al. Dietary inflammatory index and incidence of cardiovascular disease in the PREDIMED Study. Nutrients. (2015) 7:4124-38. doi: 10.3390/nu7064124

26. Vissers LE, Waller MA, van der Schouw YT, Hebert JR, Shivappa N, Schoenaker DA, et al. The relationship between the dietary inflammatory index and risk of total cardiovascular disease, ischemic heart disease and cerebrovascular disease: findings from an Australian population-based prospective cohort study of women. Atherosclerosis. (2016) 253:164-70. doi: 10.1016/j.atherosclerosis.2016.07.929

27. Shivappa N, Hebert JR, Kivimaki M, Akbaraly T. Alternative healthy eating index 2010, dietary inflammatory index and risk of mortality: results from the Whitehall II cohort study and metaanalysis of previous dietary inflammatory index and mortality studies. Br J Nutr. (2017) 118:210-21. doi: 10.1017/S00071145170 01908
28. Shivappa N, Godos J, Hebert JR, Wirth MD, Piuri G, Speciani AF, et al. Dietary inflammatory index and colorectal cancer risk-a meta-analysis. Nutrients. (2017) 9:1043. doi: 10.3390/nu9091043

29. Adoamnei E, Cutillas-Tolin A, Mendiola J, Lopez-Espin JJ, Shivappa $\mathrm{N}$, Vioque J, et al. [Associations between dietary inflammatory index and male reproductive parameters]. Rev Int Androl. (2019) 17:79-87. doi: 10.1016/j.androl.2018.03.002

30. WHO Laboratory Manual for the Examination and Processing of Human Semen. World Health Organization (2021).

31. Eslamian G, Amirjannati N, Rashidkhani B, Sadeghi MR, Hekmatdoost A. Nutrient patterns and asthenozoospermia: a case-control study. Andrologia. (2017) 49. doi: 10.1111/and.12624

32. Motrich RD, Salazar FC, Breser ML, Mackern-Oberti JP, Godoy GJ, Olivera $\mathrm{C}$, et al. Implications of prostate inflammation on male fertility. Andrologia. (2018) 50:e13093. doi: 10.1111/and.13093

33. Guo H, Xu YM, Ye ZQ, Yu JH, Fu Q, Sa YL, et al. Heat-shock protein 70 expression in the seminal plasma of patients with chronic bacterial prostatitis and chronic prostatitis/chronic pelvic pain syndrome. Prostate Cancer Prostatic Dis. (2010) 13:338-42. doi: 10.1038/pcan.2010.22

34. Marconi M, Pilatz A, Wagenlehner F, Diemer T, Weidner W. Impact of infection on the secretory capacity of the male accessory glands. Int Braz J Urol. (2009) 35:299-308, 308-9. doi: 10.1590/S1677-55382009000300006

35. Eggert-Kruse W, Kiefer I, Beck C, Demirakca T, Strowitzki T. Role for tumor necrosis factor alpha (TNF-alpha) and interleukin 1-beta (IL-1beta) determination in seminal plasma during infertility investigation. Fertil Steril. (2007) 87:810-23. doi: 10.1016/j.fertnstert.2006.08.103

36. West DW, Schuman KL, Lyon JL, Robison LM, Allred R. Differences in risk estimations from a hospital and a population-based case-control study. Int J Epidemiol. (1984) 13:235-9. doi: 10.1093/ije/13.2.235

37. D’Avanzo B, La Vecchia C, Katsouyanni K, Negri E, Trichopoulos D. An assessment, and reproducibility of food frequency data provided by hospital controls. Eur J Cancer Prev. (1997) 6:288-93. doi: 10.1097/00008469-199706000-00006

38. D' AB, La Vecchia C, Katsouyanni K, Negri E, Trichopoulos D. Reliability of information on cigarette smoking and beverage consumption provided by hospital controls. Epidemiology. (1996) 7:312-5. doi: 10.1097/00001648-199605000-00018

39. Ruiz-Canela M, Zazpe I, Shivappa N, Hebert JR, Sanchez-Tainta A, Corella $\mathrm{D}$, et al. Dietary inflammatory index and anthropometric measures of obesity in a population sample at high cardiovascular risk from the PREDIMED (PREvencion con DIeta MEDiterranea) trial. Br J Nutr. (2015) 113:984-95. doi: 10.1017/S0007114514004401

40. Nobles CJ, Schisterman EF, Ha S, Kim K, Mumford SL, Buck LG, et al. Ambient air pollution and semen quality. Environ Res. (2018) 163:228-36. doi: 10.1016/j.envres.2018.02.004

Conflict of Interest: The authors declare that the research was conducted in the absence of any commercial or financial relationships that could be construed as a potential conflict of interest.

Publisher's Note: All claims expressed in this article are solely those of the authors and do not necessarily represent those of their affiliated organizations, or those of the publisher, the editors and the reviewers. Any product that may be evaluated in this article, or claim that may be made by its manufacturer, is not guaranteed or endorsed by the publisher.

Copyright (c) 2021 Liu, Wang, Wen, Wang, Zhang, Zhang, Jiang, Zhang, Sun, Pan and $W u$. This is an open-access article distributed under the terms of the Creative Commons Attribution License (CC BY). The use, distribution or reproduction in other forums is permitted, provided the original author(s) and the copyright owner(s) are credited and that the original publication in this journal is cited, in accordance with accepted academic practice. No use, distribution or reproduction is permitted which does not comply with these terms. 